\title{
Altered Intrinsic Connectivity Networks in Frontal Lobe Epilepsy: A Resting-State fMRI Study
}

\author{
Xinzhi Cao, ${ }^{1,2}$ Zhiyu Qian, ${ }^{1}$ Qiang Xu, ${ }^{2}$ Junshu Shen, ${ }^{3}$ \\ Zhiqiang Zhang, ${ }^{2}$ and Guangming $\mathrm{Lu}^{1,2}$ \\ ${ }^{1}$ Department of Biomedical Engineering, Nanjing University of Aeronautics and Astronautics, Nanjing 210016, China \\ ${ }^{2}$ Department of Medical Imaging, Jinling Hospital, Medical School of Nanjing University, Nanjing 210002, China \\ ${ }^{3}$ Department of Radiotherapy, Jinling Hospital, Medical School of Nanjing University, Nanjing 210002, China
}

Correspondence should be addressed to Zhiyu Qian; zhiyu@nuaa.edu.cn and Guangming Lu; cjr.luguangming@vip.163.com

Received 21 July 2014; Revised 12 October 2014; Accepted 2 November 2014; Published 26 November 2014

Academic Editor: Valeri Makarov

Copyright (C) 2014 Xinzhi Cao et al. This is an open access article distributed under the Creative Commons Attribution License, which permits unrestricted use, distribution, and reproduction in any medium, provided the original work is properly cited.

\begin{abstract}
Examining the resting-state networks (RSNs) may help us to understand the neural mechanism of the frontal lobe epilepsy (FLE). Resting-state functional MRI (fMRI) data were acquired from 46 patients with FLE (study group) and 46 age- and gender-matched healthy subjects (control group). The independent component analysis (ICA) method was used to identify RSNs from each group. Compared with the healthy subjects, decreased functional connectivity was observed in all the networks; however, in some areas of RSNs, functional connectivity was increased in patients with FLE. The duration of epilepsy and the seizure frequency were used to analyze correlation with the regions of interest (ROIs) in the nine RSNs to determine their influence on FLE. The functional network connectivity (FNC) was used to study the impact on the disturbance and reorganization of FLE. The results of this study may offer new insight into the neuropathophysiological mechanisms of FLE.
\end{abstract}

\section{Introduction}

As the second highest type of localization-related epilepsies, frontal lobe epilepsy (FLE) accounts for 20 to 30 percent among all partial epilepsies [1]. However, compared with temporal lobe epilepsy (TLE), FLE in general has been less studied. Seizures in patients with FLE are varied and can be divided into perirolandic, supplementary sensorimotor area, dorsolateral, orbitofrontal, anterior frontopolar, opercular, and cingulate types [2]. Seizures originating from the perirolandic and supplementary sensorimotor areas can be distinguished, by the manifestation of motor activity or asymmetric tonic posturing with preserved awareness. However, seizures arising from dorsolateral, orbitofrontal, frontopolar, and cingulate areas have more variable clinical manifestations, and they cannot be distinguished from each other easily. The seizures frequently occur during sleep, without warning, are very short, and are followed by a rapid recovery [3].
Except for the obvious abnormalities, such as previous brain trauma, neoplasms, vascular malformations, and developmental lesions, no clear cause has been found in most patients with FLE. Furthermore, regular structure MRI imaging shows nothing valuable. However, the mechanism of how the epilepsy focus influences the functional network and further leads to the deficits of the brain's function and cognitive behavior is still not clear. Contemporary fMRI has provided effective approaches for measuring the functional alteration caused by epilepsy in brain, which is a fourdimensional medical imaging modality that captures changes in blood oxygenation over time, an indirect measure of neuronal activation.

Although EEG-fMRI and resting-state fMRI are commonly used in the study of epilepsy $[4,5]$, scalp recording of EEG changes in FLE often had the poor spatial resolution and discharge localization [6]. Resting-state fMRI is a powerful technique for the exploring of abnormal intrinsic activity 
caused by epilepsy in the brain, with good spatial and temporal resolution. ICA is a powerful method to investigate the resting-state fMRI data. As a blind source separation method, it can recover a set of signals from their linear mixtures and has yielded fruitful results with fMRI data [7]. Spatial ICA decomposes fMRI data into a set of maximally spatially independent maps and their corresponding time courses [8]. The dynamics of the blood oxygen level dependent (BOLD) signal within a single component is described by that component's time courses in resting-state fMRI data. Regions contributing significantly within a given component are strongly functionally connected to each other. This kind of biological relevance is known as resting-state networks (RSNs) [9]. RSNs are used as a robust tool to the intrinsic functional architecture of the human brain for their high reproducibility and moderate to high test-retest reliability [10].

Most studies have focused on the RSNs used with restingstate fMRI in epilepsy. Widjaja et al. showed impaired default mode network (DMN) in children with medically refractory epilepsy [11]. Zhang et al. found dorsal attention network (DAN) and auditory network (AN) impaired in temporal lobe epilepsy (TLE) $[12,13]$. Woodward et al. considered functional connectivity decreased in the motor network [14], and they all concluded that the motor network had been impaired. Wang et al. found functional connectivity decreased in the self-referential network (SRN), somatomotor network (SMN), and visual network (VN); they also found both decreased and increased in the DMN and DAN in patients with generalized tonic-clonic seizures (GTCS) [15]. Luo et al. presented reduction of functional connectivity in SMN when using group ICA on resting-state fMRI data [16].

Amann et al. extracted ROIs, regions of altered functional connectivity in brain, from RSNs and used $z$-scores obtained from ICA analysis for correlation analysis [17]. Braakman et al. found the ROIs of the RSNs were related to epilepsy factors including duration of epilepsy and seizure frequency [18]. These results may be useful to understand the influence of epilepsy with those clinical variables.

However, these studies were all focused on single restingstate functional network. Little attention was paid to the interactions between the RSNs in patients with epilepsy. Luo et al. implicated that investigating the interactions between the RSNs might be helpful to understand the neuropathophysiological mechanism of epilepsy globally [16]. By using functional network connectivity (FNC), they found that the lost interactions among the intersystem might be associated with the disturbance of the high level complex function which is needed in the integrated multisystems of partial epilepsy. The FNC toolbox calculated the time lags between components correlated with each other, and FNC maps were constructed via a partial correlation analysis of ICA time courses. This approach has been published and applied to the identification of which pairs of RSNs show changes in temporal correlation between patients and healthy controls (HC) [19].

FLE may involve multiple interacting networks, and few studies have focused on analyzing the functional connectivity of RSNs in patients with FLE and FNC relationships.
Investigations on RSNs in patients with FLE could provide valuable data to validate the hypothesis that the abnormal neuroactivity generated from epilepsy focus leads to functional network impairment. The primary aim of the present work was to determine whether the intranetwork of RSNs might be aberrant in patients with FLE on resting-state fMRI data. The study also was concerned about the significant correlation between altered ROIs of the RSNs and clinical variables, including seizure frequency and the duration of epilepsy. Examination of the differences in FNC between the patients with FLE and $\mathrm{HC}$ may be helpful to understand the impact on the disturbance and reorganization of FLE. The results of this study may provide new insight into the neuropathophysiological mechanisms of FLE.

\section{Material and Methods}

2.1. Participants. Patients with FLE were recruited from a patient population who had received clinical treatments in Jinling Hospital. Forty-six healthy subjects were selected as the control group that matches the age and gender of the FLE group. Two-sample $t$-test of age showed $P>0.05$, and the result of Pearson chi-square test of gender showed $P>0.05$. Clinical information and demographic of patients with FLE and $\mathrm{HC}$ are shown in Table 1.

Patients with FLE were diagnosed based on the International League against Epilepsy (ILAE) classification [20]. Moreover, all the patients reached the inclusive criteria as follows: (1) one or more typical symptoms of FLE, including night seizure or posturing; (2) during electrophysiological monitoring, ictal EEG demonstrated typical generalized epileptiform spikes; (3) predominantly interictal epileptic discharges were shown by scalp EEGs; (4) there was no abnormality in the images of regular structural MRI. All subjects gave their written informed consent to participate in this study, which had been approved by the Medical Ethics Committee in Jinling Hospital.

2.2. Data Acquisition. Functional MRI data were acquired on a Magnetom Trio 3T MR Scanner (Siemens AG, Erlangen, Germany). During the resting-state fMRI session, the participants were instructed to relax with their eyes closed and keep their heads still during the scans. Functional images were subsequently acquired in the same slice orientation with a GRE-EPI (gradient recalled echo, echo-planar imaging) sequence $\left(\mathrm{TR} / \mathrm{TE}=2,000 \mathrm{~ms} / 30 \mathrm{~ms}\right.$, FOV $24.0 \times 24.0 \mathrm{~cm}^{2}, \mathrm{FA}$ $90^{\circ}$, matrix $64 \times 64$, slice thickness $4.0 \mathrm{~mm}$, slice gap $0.4 \mathrm{~mm}$, 30 slices, acquisition voxel size $=3.0 \times 3.0 \times 3.0 \mathrm{~mm}$ ) for eight minutes and twenty seconds (250 measurements). 3D T1weighted images were also acquired by using a 3DMPRAGE sequence, matrix $256 \times 256$, slice thickness $1.0 \mathrm{~mm}$.

2.3. fMRI Data Preprocessing. A SPM- (statistical parametric mapping-) based fMRI data processing pipeline DPARSF (http://www.restfmri.net/forum/) was used to perform the data preprocessing, including the slice timing correction and motion correction. Data was excluded if the head motion exceeded $1.0 \mathrm{~mm}$ or if rotation exceeded $1.0^{\circ}$ during scanning. 
TABLE 1: Clinical information and demographic of patients with FLE and HC.

\begin{tabular}{|c|c|c|c|c|c|}
\hline Groups & Men & $\begin{array}{c}\text { Age } \\
\text { (years) }\end{array}$ & $\begin{array}{c}\text { Duration } \\
\text { (years) }\end{array}$ & $\begin{array}{l}\text { Seizure frequency } \\
\text { (times per year) }\end{array}$ & Treatment \\
\hline $\operatorname{FLE}(n=46)$ & 21 & $\begin{array}{c}26 \pm 4.59 \\
(19-35)\end{array}$ & $\begin{array}{l}9.2 \pm 7.82 \\
\quad(1-27)\end{array}$ & $\begin{array}{c}59.66 \pm 166.67 \\
(0.42-912)\end{array}$ & $\begin{array}{l}\text { Carbamazepine: } 11, \\
\text { topiramate: } 5 \text {, } \\
\text { oxcarbazepine: } 3 \text {, } \\
\text { phenobarbital: } 6 \text {, } \\
\text { sodium valproate: } 16 \text {, } \\
\text { no treatment: } 5\end{array}$ \\
\hline $\mathrm{HC}(n=46)$ & 22 & $\begin{array}{c}25.3 \pm 5.33 \\
(20-39)\end{array}$ & I & I & I \\
\hline
\end{tabular}

The 3D T1-weighted images were used for normalizing the fMRI images after being resampled to $3.0 \mathrm{~mm} \times 3.0 \mathrm{~mm}$ $\times 3.0 \mathrm{~mm}$. After being smoothed with a full width at half maximum (FWHM) of $8.0 \mathrm{~mm}$, the $\mathrm{fMRI}$ data were prepared for further processing.

2.4. RSNs Extraction Using ICA. Group spatial ICA was conducted by using the infomax algorithm with the GIFT software (http://icatb.sourceforge.net/) in Matlab (The MathWorks Inc.). The fMRI data of the FLE and HC group were calculated to identify intrinsic networks, by estimating maximally independent spatial sources among ninety-two subjects. The data of all 92 subjects were decomposed into 29 independent components (ICs). As a preprocessing step to ICA decomposition, the principal component analysis (PCA) was conducted to reduce data dimensions. Single subject time courses and spatial maps were then back-reconstructed. The relatively high model order ICA was chosen, which could yield refined components [21]. The eight ICs for further analysis were selected based on the largest spatial correlation [22] with specific RSN templates reported in previous studies [2325], which correspond to known anatomical and functional segmentations. The RSN templates are shown in Figure 1. These were done by using BrainNet Viewer [26] and included core network $(\mathrm{CN})$, default mode network (anterior DMN, posterior DMN), central executive network (CEN), somatomotor network (SMN), self-referential network (SRN), dorsal attention network (DAN), auditory network (AN), and visual network (VN).

2.5. Intranetwork Analysis. Group comparisons were restricted to the voxels within each corresponding RSN. Two-sample $t$-test $(P<0.05$, AlphaSim corrected) was employed for group comparisons for the areas within the mask of each RSN. The mask for group comparisons was created by combining the regions of the RSN by using one-sample $t$-test $(P<0.05$, AlphaSim corrected) [27] among the ICs marked as the same RSNs of each individual subject in both the patients and controls [28].

The effects of clinical variables including seizure frequency and the duration of epilepsy were analyzed in correlation with the alterations in RSNs in epilepsy. From each corresponding RSN, the voxels showing significant differences (positive or negative) between the patient and the control groups were extracted as a mask consisting of several regions of interest (ROIs). The mean $\mathrm{z}$-scores, obtained from ICA analysis, within each mask were correlated with the seizure frequency and the duration of epilepsy, which is considered to reflect the influence of the disease.

2.6. Internetwork Analysis. The ICA algorithm assumed that the time courses of cortical areas within one component were synchronous. Though the components were spatially independent, significant temporal correlations could exist between them. As an extension of ICA analysis, the FNC toolbox (http://mialab.mrn.org/software/\#fnc) was employed to examine the temporal relationships between brain networks. Corresponding to the significant correlation combinations, the average time lags, which represented the amount of delay between two correlated RSNs' time courses, were calculated for each group. The maximum time lag was set to $3 \mathrm{~s}$ [29]. One-sample $t$-test $(P<0.05$, FDR corrected) for each group and two-sample $t$-test $(P<0.05$, FDR corrected) for group comparisons were performed on all possible combinations [16].

\section{Results}

3.1. Intranetwork Alterations of RSNs in FLE. Since the DMN was split into aDMN and pDMN, nine resting-state networks (RSNs) were identified by using eight templates in all 29 ICA components for each of the 92 subjects. Correlation coefficients between the spatial templates and ICs of ICA analysis were as follows: CN (IC03), 0.297; aDMN (IC06), 0.488; CEN (IC07), 0.341; SMN (IC11), 0.755; SRN (IC18), 0.367; DAN (IC23), 0.511; AN (IC25), 0.783; VN (IC27), 0.771; pDMN (IC28), 0.383. The results of the one-sample $t$-test $(P<0.05$, AlphaSim corrected $)$ in the patients with FLE and $\mathrm{HC}$ are shown in Figure 2 by using BrainNet Viewer.

The two-sample $t$-test $(P<0.05$, AlphaSim correlated) revealed the differences in functional connectivity within each RSN between the two groups. Compared with the controls, decreased functional connectivity (negative $T$ value) was found in all the nine networks and increased functional connectivity (positive $T$ value) was found in some of them including aDMN, VN, and pDMN. Voxels above the threshold were picked up. Within each area, the maximum $T$ value and its MNI coordinate were provided. The threshold 


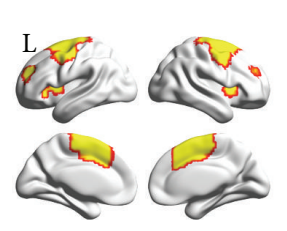

$\mathrm{CN}$

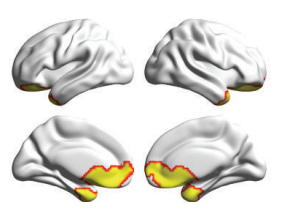

SRN

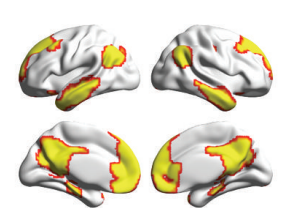

DMN

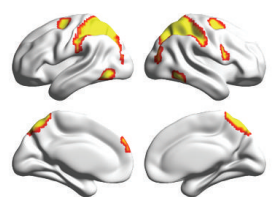

DAN

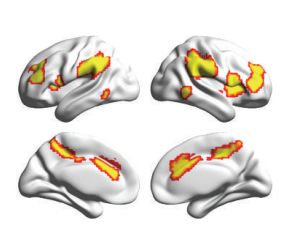

CEN

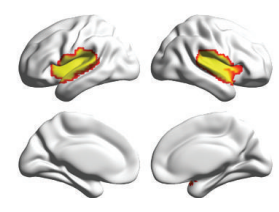

$\mathrm{AN}$

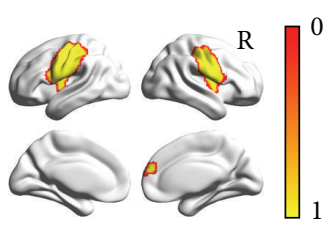

SMN

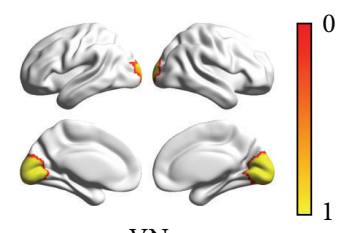

$\mathrm{VN}$

FIGURE 1: Eight templates of RSNs for estimating maximally independent spatial components among ninety-two subjects, including CN, DMN, CEN, SMN, SRN, DAN, AN, and VN, shown by BrainNet Viewer. The T value range is 0-1.

of cluster size in each RSN was also offered according to the AlphaSim correlation, as shown in Table 2 and Figure 3 by using BrainNet Viewer.

Significant negative correlations were shown between the mean $z$-scores of ROIs extracted from altered RSNs and the seizure frequency in CEN (cingulate gyrus, voxels $=118, P=$ 0.0162 , and $r=-0.353$ ), AN (Frontal_Mid_R, voxels $=41$, $P=0.017$, and $r=-0.35$ ), and VN (Calcarine_R, voxels $=35, P=0.0296$, and $r=-0.321)$ in Figure 4(a). On the other hand, the significant negative correlations between the mean $z$-scores and the duration of epilepsy were found in CN (Frontal_Sup_Medial_L, voxels $=35, P=0.0136$, and $r=$ -0.362 ) and SMN (Precentral_R, voxels $=56, P=0.000687$, and $r=-0.482$ ), as shown in Figure 4(b).

3.2. Internetwork Alterations of RSNs in FLE. Significant correlation combinations were extracted for both groups separately and the results of FNC maps for each group are shown in Figure 5. It was found that 28 out of the 36 possible combinations were significant in FLE group (Figure 5(a)) and 27 out of the 36 possible combinations were significant in the HC group (Figure 5(b)). No significant difference $(P<0.05$, FDR corrected) was found in each combination between FLE and HC group. Arrows represent a significant correlation between RSNs $(P<0.05$, FDR corrected). For example, an arrow that connected the $\mathrm{CN}$ and aDMN (pointing toward the latter in the FLE group) signifies that significant correlation was obtained between them. Its pointing direction and the color of the arrow indicated the time lag from $\mathrm{CN}$ to $\mathrm{aDMN}$.

The FNC results of the FLE group showed alterations in inter-RSNs connectivity; the combinations and the time lags were altered in the pairs of RSNs. Compared to the controls, combinations were found to be lost between aDMN/SMN, $\mathrm{CN} / \mathrm{SRN}, \mathrm{CN} / \mathrm{pDMN}$, and SMN/pDMN, while combinations were found to be newly added between $\mathrm{CN} / \mathrm{aDMN}$, $\mathrm{CEN} / \mathrm{aDMN}, \mathrm{CEN} / \mathrm{SRN}, \mathrm{CEN} / \mathrm{VN}$, and AN/pDMN in the FLE group. Time lags were found lower between CN/SMN but higher between DAN/SMN than in the HC group. Also observed were the directional differences in the time lags among components (i.e., from $\mathrm{VN}$ to $\mathrm{CN}$ in patients, while from $\mathrm{CN}$ to $\mathrm{VN}$ in controls).

\section{Discussion}

This study focused on the intranetwork alterations of the RSNs in the FLE. Nine RSNs were selected to be analyzed. Compared with the HC group, decreased functional connectivity was observed in all the nine RSNs; however, in some areas of RSNs, functional connectivity was increased, including aDMN (Cingulum_Mid_L, Precuneus_R), VN (lingual gyrus), and pDMN (Postcentral_R, Precuneus_R). Correlation analysis found significant negative correlation between seizure frequency and CEN (ROI in cingulate gyrus), AN (ROI in Frontal_Mid_R), and VN (ROI in Calcarine_R) and significant negative correlation between duration of epilepsy and CN (ROI in Frontal_Sup_Medial_L), SMN (ROI in Precentral_R). Compared with the HC group, alterations of combinations in the intersystem of the RSNs were determined by FNC in the FLE group, but no significant difference was found between the FLE group and the HC group.

4.1. Intranetwork Alterations. DMN, hypothesized to be involved in cognitive functions associated with intrinsic processing and external inputs [30], was split into the anterior areas $(\mathrm{aDMN})$ and the posterior areas $(\mathrm{pDMN})$ in the current study [31]. DMN involves PCC/PCUN, bilateral inferior parietal gyrus, angular gyrus, middle temporal gyrus, superior frontal gyrus, and medial frontal gyrus [25]. Danielson et al. found activity decreased in DMN in epilepsy by using a wide range of neuroimaging and electrophysiological modalities, during complex partial, generalized tonic-clonic, and absence of seizures [32]. They supported network inhibition hypothesis that active inhibition of arousal systems by seizures in certain cortical regions leads to cortical deactivation in other cortical areas. Many studies [33, 34] also presented the significant reduced functional connectivity in DMN in epilepsy patients, including the aDMN and the pDMN. They concluded that it might momentarily reduce the consciousness level and cognitive reserve. Liao et al. found 

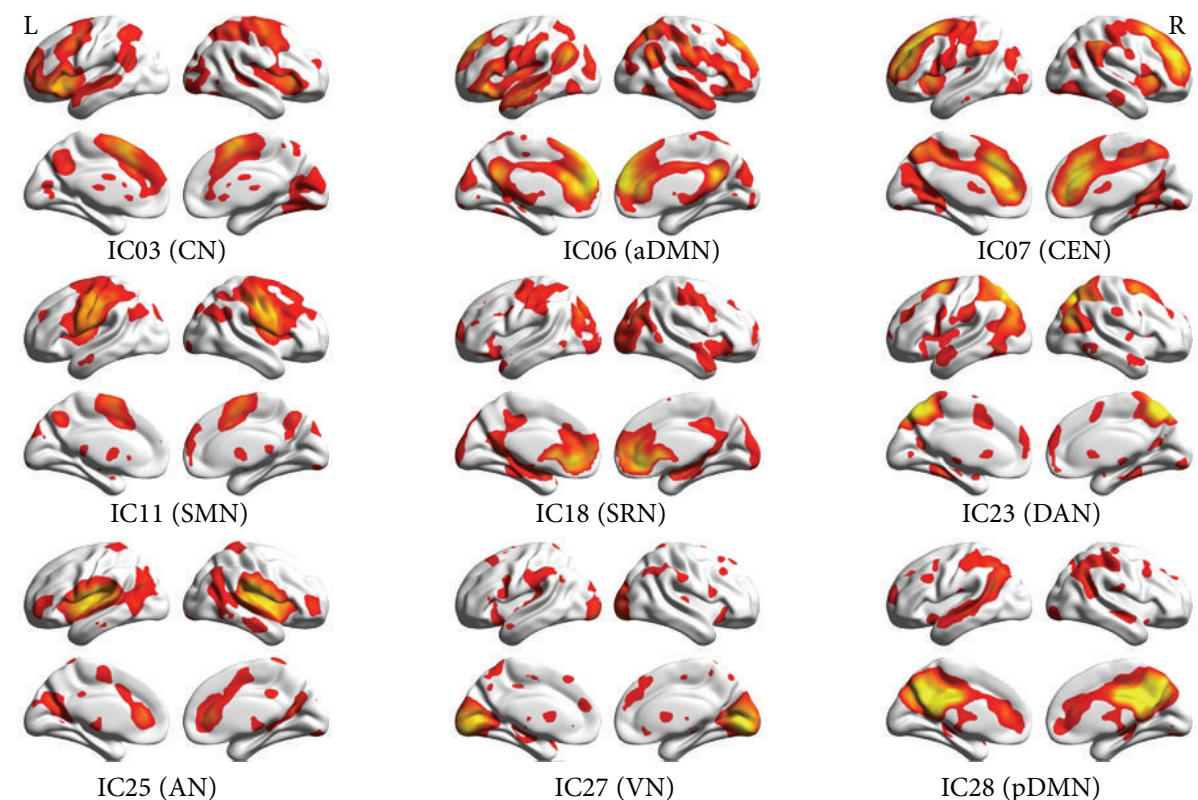

IC07 (CEN)
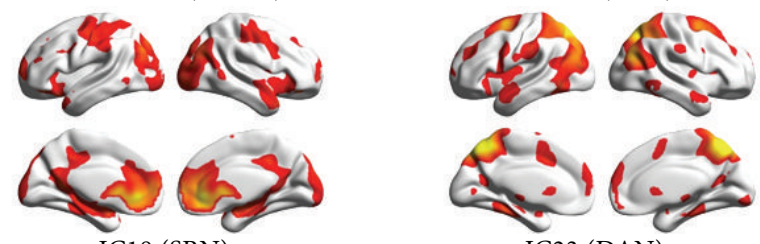

IC18 (SRN)

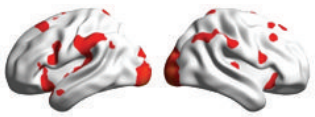

IC23 (DAN)
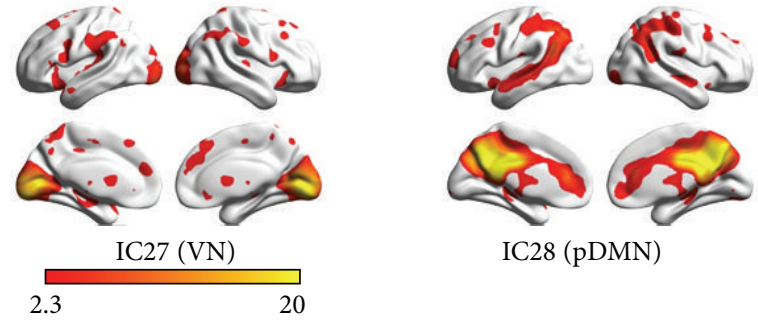

IC28 (pDMN)

(a)

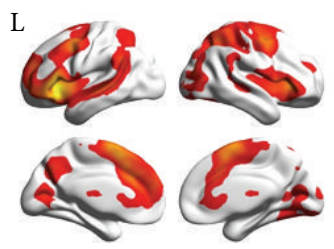
IC03 (CN)
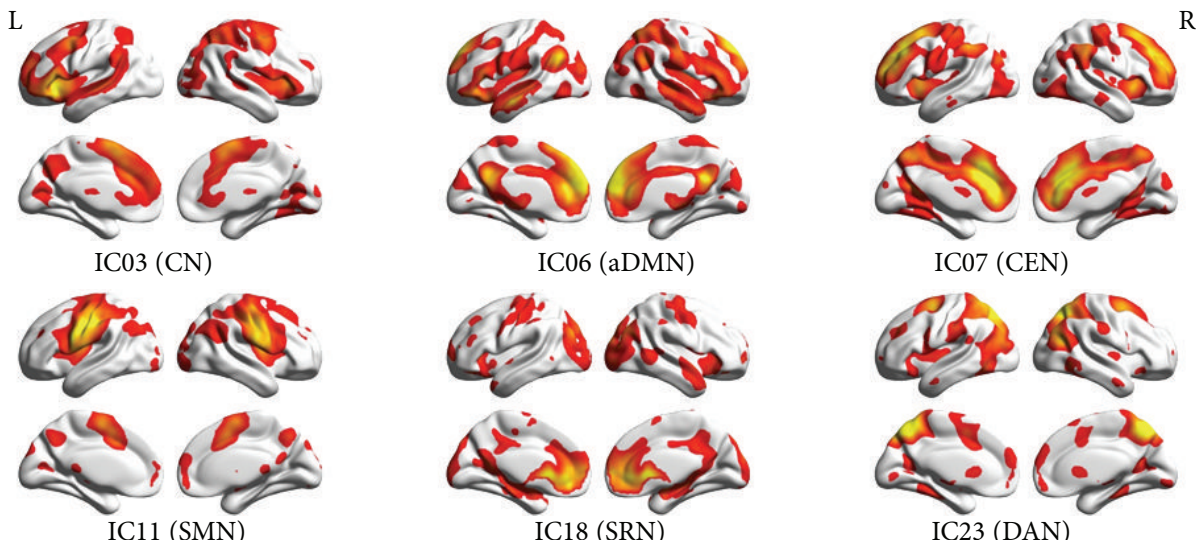

IC06 (aDMN)

IC07 (CEN)
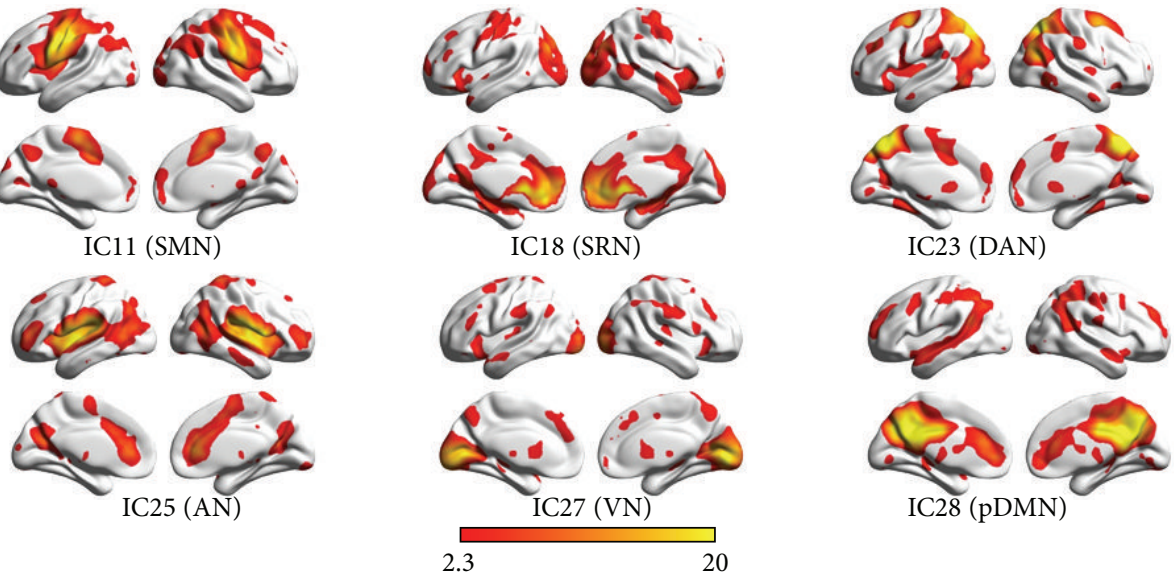

(b)

Figure 2: Results of one-sample $t$-test $(P<0.05$, AlphaSim corrected) in nine RSNs of FLE (a) and HC (b), shown by BrainNet Viewer. The $T$ value range is $2.3-20$.

functional and structural connectivity decreased in aDMN and $\mathrm{pDMN}$ by using functional and structural (path length and connection density derived from DTI tractography) MRI in mTLE [35], but Zhang et al. found functional connectivity increased in the pDMN, suggesting that the posterior cingulate cortex might play a compensatory role for the altered DMN in the right mTLE [36]. In the present study, decreased functional connectivity of the DMN may implicate cognitive impairment, and the increased functional connectivity of the pDMN may play a compensatory role in patients with FLE. One interpretation was that the pDMN was involved in initiation of spike and slow-wave discharges activity [15].

In the perceptual network, SMN includes pre- and postcentral gyrus, the primary sensory-motor cortices, and 


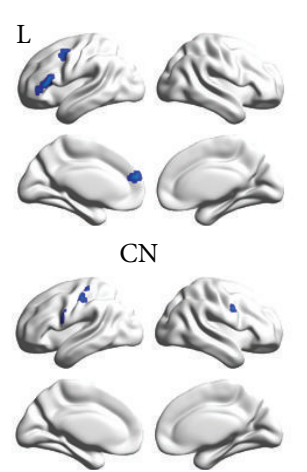

SMN
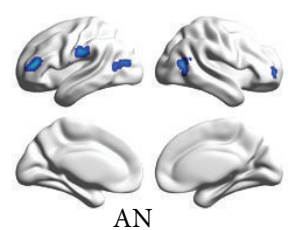

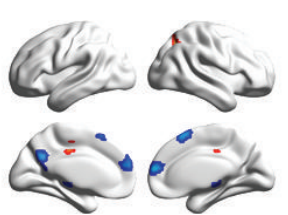

$\mathrm{aDMN}$

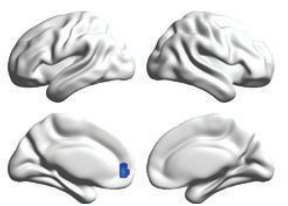

SRN

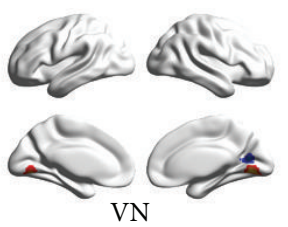

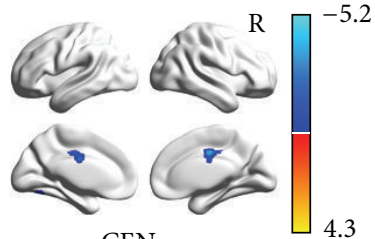

CEN

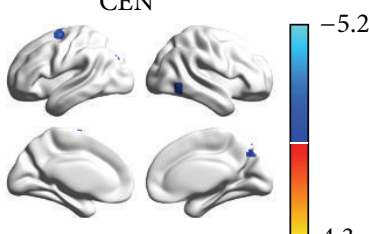

DAN

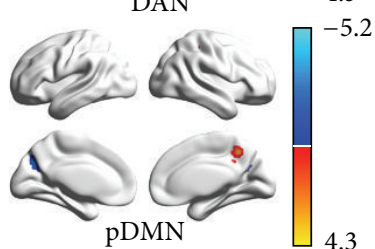

Figure 3: Results of the two-sample $t$-test $(P<0.05$, AlphaSim correlated) of the RSNs between the patients with FLE and HC, shown by BrainNet Viewer. Decreased functional connectivity was observed (negative $T$ value, maximum peak $T=-5.10$ ) in all the nine networks and increased (positive $T$ value, maximum peak $T=4.29$ ) functional connectivity was observed in some areas of them including aDMN, $\mathrm{VN}$, and $\mathrm{pDMN}$.
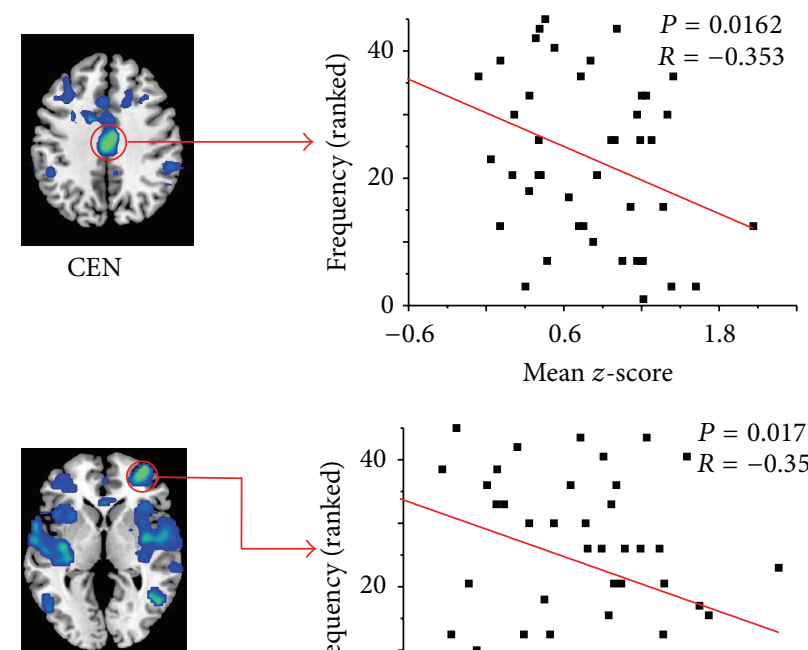

AN
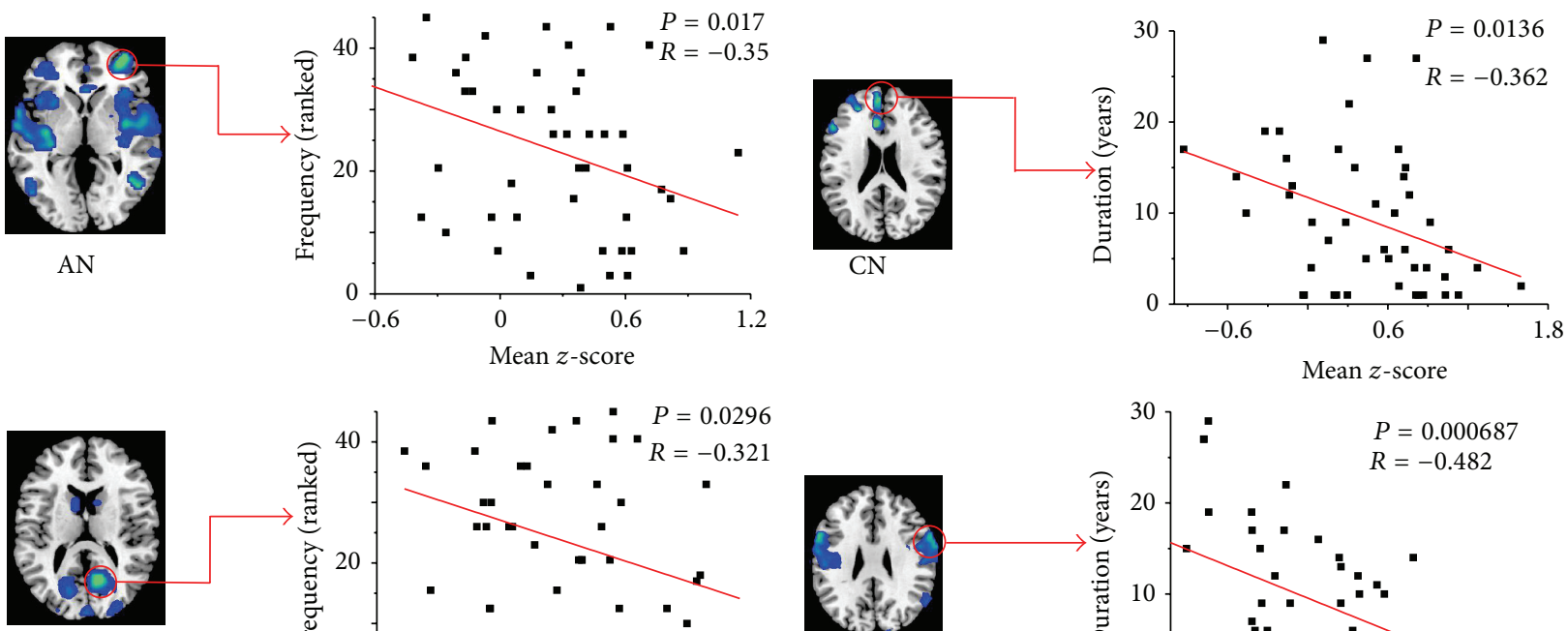

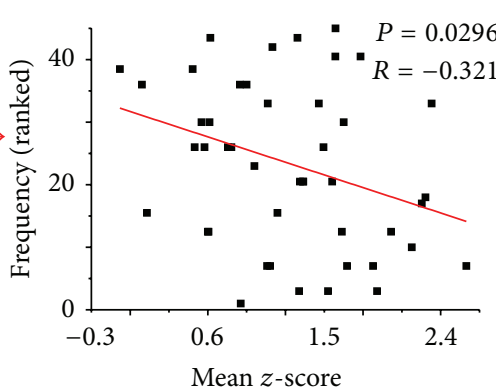

(a)

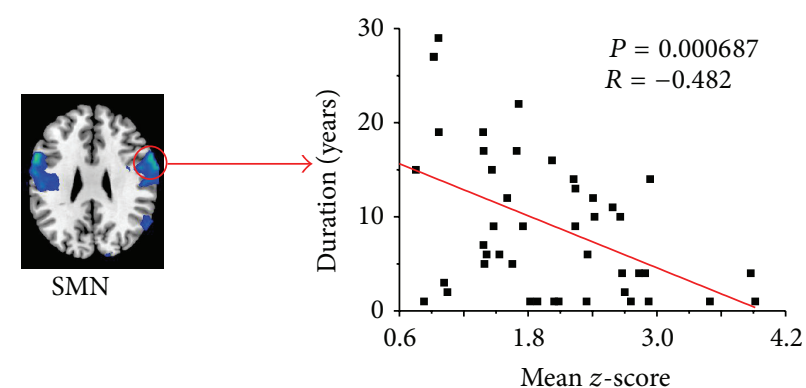

(b)

FIGURE 4: Significant negative correlations between the mean $z$-scores of ROIs and the epilepsy frequency (ranked) in CEN, AN, and VN are shown in (a). Significant negative correlations between the mean $z$-scores of ROIs and duration of epilepsy in CN and SMN are shown in (b). 
TABLE 2: Group comparisons of the nine RSNs between the patients with FLE and HC.

\begin{tabular}{|c|c|c|c|}
\hline Brain regions & $\begin{array}{l}\text { Coordinates } \\
x, y, z(\mathrm{MNI})\end{array}$ & $T$ value (peak) & Voxels \\
\hline \multicolumn{4}{|l|}{$\mathrm{CN}$ (cluster size $>33$ ) } \\
\hline Frontal_Inf_Tri_L & -512418 & -3.55 & 96 \\
\hline Frontal_Sup_Medial_L & -35721 & -3.27 & 35 \\
\hline Precentral_L & -39348 & -3.29 & 34 \\
\hline \multicolumn{4}{|l|}{ aDMN (cluster size $>36)$} \\
\hline Frontal_Sup_Medial_L & 95715 & -4.54 & 172 \\
\hline Superior frontal gyrus & 31857 & -3.71 & 71 \\
\hline Precuneus_L & $-3-6333$ & -3.90 & 67 \\
\hline Angular_L & $-39-5730$ & -5.10 & 48 \\
\hline Cingulum_Mid_L & $-6-2442$ & 4.19 & 38 \\
\hline Precuneus_R & $21-6342$ & 3.61 & 37 \\
\hline \multicolumn{4}{|l|}{ CEN $($ cluster size $>43)$} \\
\hline Cingulate gyrus & $6-1239$ & -3.87 & 118 \\
\hline \multicolumn{4}{|l|}{ SMN (cluster size $>34$ ) } \\
\hline Precentral_L & -60924 & -3.19 & 56 \\
\hline Precentral_R & 60933 & -2.79 & 56 \\
\hline Postcentral_L & $-42-2466$ & -3.66 & 52 \\
\hline \multicolumn{4}{|l|}{ SRN (cluster size $>26)$} \\
\hline Medial frontal gyrus & -3510 & -3.66 & 69 \\
\hline \multicolumn{4}{|l|}{ DAN (cluster size $>35)$} \\
\hline Temporal_Inf_R & $48-60-6$ & -4.62 & 37 \\
\hline Frontal_Sup_L & $-18-363$ & -4.04 & 44 \\
\hline Occipital_Mid_L & $-24-7230$ & -3.34 & 42 \\
\hline Precuneus_R & $0-7851$ & -3.82 & 49 \\
\hline \multicolumn{4}{|l|}{ AN (cluster size > 36) } \\
\hline Frontal_Inf_Tri_L & -393912 & -4.97 & 69 \\
\hline Frontal_Mid_R & 36570 & -4.32 & 41 \\
\hline Temporal_Mid_R & $42-6012$ & -3.63 & 84 \\
\hline Temporal_Mid_L & $-42-639$ & -3.60 & 50 \\
\hline Postcentral_L & $-63-2127$ & -3.87 & 104 \\
\hline \multicolumn{4}{|l|}{ VN (cluster size $>34$ ) } \\
\hline Lingual gyrus & $15-690$ & 4.29 & 186 \\
\hline Calcarine_R & $12-6315$ & -3.64 & 35 \\
\hline \multicolumn{4}{|l|}{ pDMN (cluster size $>36)$} \\
\hline Postcentral_R & $27-3042$ & 4.07 & 68 \\
\hline Precuneus_R & $18-4845$ & 3.71 & 70 \\
\hline Precuneus_L & $-15-6633$ & -4.41 & 52 \\
\hline Precuneus_R & $21-6330$ & -3.55 & 41 \\
\hline
\end{tabular}

Note: $P<0.05$, AlphaSim corrected.

the supplementary motor area; AN primarily encompasses the bilateral middle and superior temporal gyrus, Heschl gyrus, and temporal pole; VN includes the inferior, middle, and superior occipital gyrus and the temporal-occipital regions along with superior parietal gyrus [25]. Zhang et al. [36] found functional connectivity decreased in perceptual networks including SMN and AN with mTLE by using fMRI. They also found functional connectivity increased in the primary visual cortex and functional connectivity decreased in the bilateral MT+ areas of the VN. They considered that the primary visual function was not impaired and there might be deficits in the high-order visual function in mTLE. Grant et al. [37] also considered that the primary visual function was not impaired in mTLE. Woodward et al. [14] and Yang et al. [38] found patients with FLE exhibited decreased functional connectivity within the motor network. Haneef et al. [39] found reduced connectivity involving areas of the sensorimotor cortex (visual, somatosensory, auditory, 


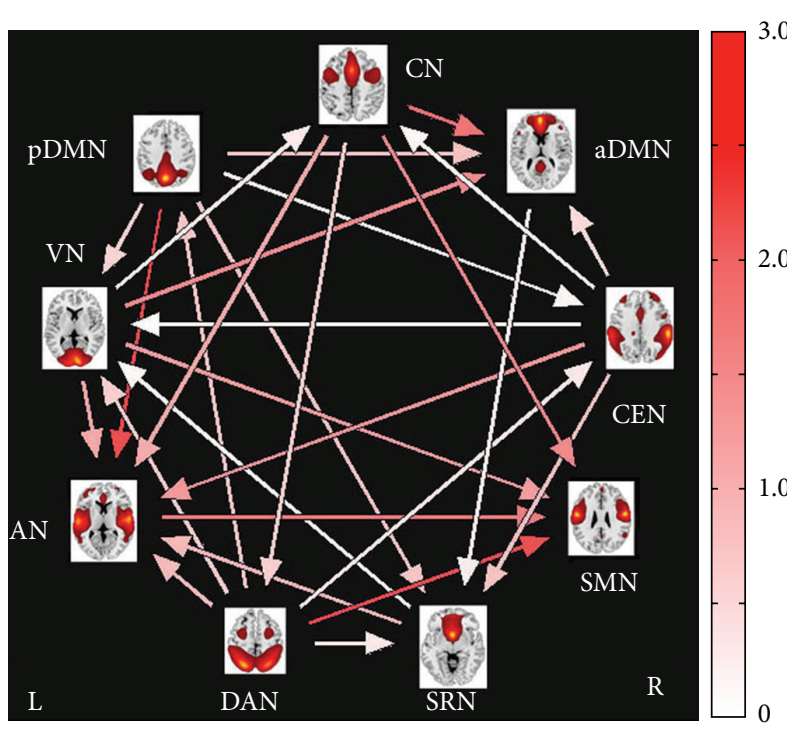

(a)

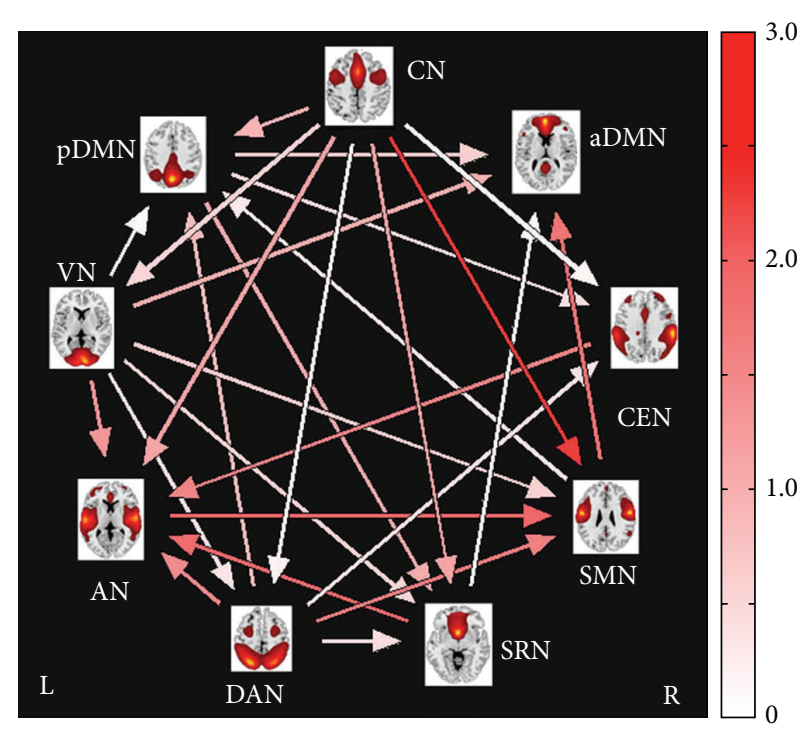

(b)

FIGURE 5: FNC results of the FLE group (a) and the HC group (b); the color bars represent time lag (0-3 s). Arrows represented a significant correlation between RSNs $(P<0.05$, FDR corrected); 28 combinations were significant in FLE group and 27 combinations were significant in the HC group.

and primary motor) in TLE. This study's work presented consistent results in FLE with previous findings in FLE or mTLE.

In this present study, functional connectivity was found to be decreased in the $\mathrm{CN}, \mathrm{CEN}$, and DAN. It might present impairments in the three networks. CN (insula-cingulate cortices) had an important impact on cognitive control, including the anterior cingulate, the bilateral insular, and dorsolateral prefrontal cortices [25]. Key regions in CEN $[40,41]$ include the dorsal lateral prefrontal cortices and the posterior parietal cortices. DAN is thought to mediate goaldirected top-down processing [25] and primarily involves the middle and superior occipital gyrus, parietal gyrus, inferior, superior parietal gyrus, middle frontal gyrus, and superior frontal gyrus. Zhang et al. also found higher order cognitive processes including CN, CEN, and DAN decreased in generalized spike-and-wave discharge during absence seizure by using EEG-fMRI [36].

The functional connectivity was found decreased in the SRN in present study. SRN comprises the ventromedial prefrontal cortex (vMPFC), medial orbital prefrontal cortex (MOPFC), gyrus rectus, and pregenual anterior cingulate gyrus (PACC) [42, 43]. Qi et al. researched the impairments of SRN in minimal hepatic encephalopathy [44], and Bai et al. [45] studied the altered pattern of SRN around amnestic mild cognitive impairment, but few studies focused on SRN in epilepsy.

Decreased functional connectivity is considered to result from the disruption of neuronal connection within a functional network and is commonly used to reflect cognitive impairments in brain disorders [46]. On the contrary, increased functional connectivity is often interpreted to reflect the enhanced functionality owing to a compensatory mechanism [47].

A higher frequency of seizures and a longer duration of epilepsy were associated with progression of gray and white matter atrophy in patients with mTLE [48]. Zhang et al. [36] found the decreased functional connectivity of the mesial temporal lobe was related to the duration of epilepsy. In this study, significant negative correlations were shown between the seizure frequency and ROIs in CEN/AN/VN. This may indicate that more impairments should be related to the higher seizure frequency in CEN (ROI in cingulate gyrus), AN (ROI in Frontal_Mid_R), and VN (ROI in Calcarine_R); significant negative correlations were found between the duration of epilepsy and ROIs in CN/SMN, and longer duration may suggest more impairments in CN (ROI in Frontal_Sup_Medial_L) and SMN (ROI in Precentral_R).

4.2. Internetwork Alterations. In this study, combinations were found to be lost and newly added in inter-RSNs connectivity compared with controls. Moreover, alterations of the time lags were found in the pairs of RSNs. Otti et al. found lost connectivities or combinations in the patients [49] with somatoform pain disorder, indicating impairments on the system level. On the contrary, Luo et al. found no significant difference of interaction in two RSNs between TLE and HC [16]. However, in this study, no significant difference of interaction in two RSNs was found in each combination between FLE and HC group. One explanation for this phenomenon was that no obvious impairment was found on the system level in FLE. Besides, the small sample size may be another reason to worsen statistical significance. 
These findings could help to understand the FLE impact on the RSNs from global perspective.

Limitation. Study limitations included a moderate sample size and mixed medication histories. Moreover, despite the fact that the present paper focused on the functions of the restingstate networks, it was likely that the regions within each component, as well as any corresponding subregions, may have functionally distinct roles. Further studies with more imaging data and simultaneous behavioral data acquisition are needed.

\section{Conclusion}

Based on the detection of the RSNs by using ICA, 29 components were extracted by ICA, and nine RSNs were selected for study. This study explored the alterations of all the nine networks in patients with FLE. The findings of decreased and increased functional connectivity may be helpful to understanding the neuropath-physiological mechanism in patients with FLE. The decreased functional connectivity of the RSNs may implicate the impairment in patients with FLE. Increased functional connectivity of the RSNs may indicate compensatory mechanism in FLE. Five significant correlations results showed that seizure frequency and duration of epilepsy have an impact on the subregions of the RSNs in patients with FLE. Moreover, this study's work is the first study that demonstrates results of resting FNC among RSNs in patients with FLE. The results may encourage further research about the effect of FLE on the human brain.

\section{Conflict of Interests}

The authors declare that there is no conflict of interests regarding the publication of this paper.

\section{Acknowledgments}

The authors would like to thank Dr. Zhang for the provision of data of patients with FLE and HC, Dr. Xu for his helpful suggestion to the paper, and Morgan for his revising of the paper. This research was supported by the Natural Science Foundation of China (Grant nos. 81422022, 81271553, $81201155,81171328,61131003,61378092$, and 81401402), Grants for Young Scholar of Jinling Hospital (Grant no. 2011061), and 12.5 Key Grant (BWS11J063 and 10z026).

\section{References}

[1] M. T. Doelken, A. Mennecke, H. J. Huppertz et al., "Multimodality approach in cryptogenic epilepsy with focus on morphometric 3T MRI," Journal of Neuroradiology, vol. 39, no. 2, pp. 87-96, 2012.

[2] R. Bagla and C. T. Skidmore, "Frontal lobe seizures," Neurologist, vol. 17, no. 3, pp. 125-135, 2011.

[3] C. P. Derry, "The sleep manifestations of frontal lobe epilepsy," Current Neurology and Neuroscience Reports, vol. 11, no. 2, pp. 218-226, 2011.
[4] M. Cosottini, I. Pesaresi, P. Maritato et al., "EEG topographyspecific BOLD changes: a continuous EEG-fMRI study in a patient with focal epilepsy," Magnetic Resonance Imaging, vol. 28, no. 3, pp. 388-393, 2010.

[5] D. An, F. Fahoum, J. Hall, A. Olivier, J. Gotman, and F. Dubeau, "Electroencephalography/functional magnetic resonance imaging responses help predict surgical outcome in focal epilepsy," Epilepsia, vol. 54, no. 12, pp. 2184-2194, 2013.

[6] L. Bianchi, L. R. Quitadamo, M. G. Marciani, B. Maraviglia, M. Abbafati, and G. Garreffa, "How the NPX data format handles EEG data acquired simultaneously with fMRI," Magnetic Resonance Imaging, vol. 25, no. 6, pp. 1011-1014, 2007.

[7] V. D. Calhoun, T. Eichele, and G. Pearlson, "Functional brain networks in schizophrenia: a review," Frontiers in Human Neuroscience, vol. 3, article 17, 2009.

[8] G. H. Turner and D. B. Twieg, "Study of temporal stationarity and spatial consistency of fMRI noise using independent component analysis," IEEE Transactions on Medical Imaging, vol. 24, no. 6, pp. 712-718, 2005.

[9] G. V. Pendse, D. Borsook, and L. Becerra, "A simple and objective method for reproducible resting state network (RSN) detection in fMRI," PLoS ONE, vol. 6, no. 12, Article ID e27594, 2011.

[10] X. N. Zuo, A. di Martino, C. Kelly et al., "The oscillating brain: complex and reliable," NeuroImage, vol. 49, no. 2, pp. 1432-1445, 2010.

[11] E. Widjaja, M. Zamyadi, C. Raybaud, O. C. Snead, and M. L. Smith, "Impaired default mode network on resting-state fMRI in children with medically refractory epilepsy," American Journal of Neuroradiology, vol. 34, no. 3, pp. 552-557, 2013.

[12] Z. Zhang, G. Lu, Y. Zhong et al., "Impaired attention network in temporal lobe epilepsy: a resting FMRI study," Neuroscience Letters, vol. 458, no. 3, pp. 97-101, 2009.

[13] Z. Zhang, G. Lu, Y. Zhong et al., "Impaired perceptual networks in temporal lobe epilepsy revealed by resting fMRI," Journal of Neurology, vol. 256, no. 10, pp. 1705-1713, 2009.

[14] K. E. Woodward, I. Gaxiola-Valdez, B. G. Goodyear et al., "Frontal lobe epilepsy alters functional connections within the brain's motor network: a resting-state fMRI study," Brain Connection, vol. 4, no. 2, pp. 91-99, 2014.

[15] Z. Wang, G. Lu, Z. Zhang et al., "Altered resting state networks in epileptic patients with generalized tonic-clonic seizures," Brain Research, vol. 1374, pp. 134-141, 2011.

[16] C. Luo, C. Qiu, Z. Guo et al., "Disrupted functional brain connectivity in partial epilepsy: a resting-state fMRI study," PLoS ONE, vol. 7, no. 1, Article ID e28196, 2012.

[17] M. Amann, J. G. Hirsch, and A. Gass, "A serial functional connectivity MRI study in healthy individuals assessing the variability of connectivity measures: reduced interhemispheric connectivity in the motor network during continuous performance," Magnetic Resonance Imaging, vol. 27, no. 10, pp. 1347$1359,2009$.

[18] H. M. H. Braakman, D. M. Ijff, M. J. Vaessen et al., "Cognitive and behavioural findings in children with frontal lobe epilepsy," European Journal of Paediatric Neurology, vol. 16, no. 6, pp. 707$715,2012$.

[19] M. J. Jafri, G. D. Pearlson, M. Stevens, and V. D. Calhoun, "A method for functional network connectivity among spatially independent resting-state components in schizophrenia," Neurolmage, vol. 39, no. 4, pp. 1666-1681, 2008. 
[20] G. Hirose, "An overview of epilepsy: its history, classification, pathophysiology and management," Brain and Nerve, vol. 65, no. 5, pp. 509-520, 2013.

[21] Q. Yu, J. Sui, S. Rachakonda et al., "Altered topological properties of functional network connectivity in schizophrenia during resting state: a small-world brain Network study," PLoS ONE, vol. 6, no. 9, Article ID e25423, 2011.

[22] V. D. Calhoun, T. Adali, G. D. Pearlson, and J. J. Pekar, "A method for making group inferences from functional MRI data using independent component analysis," Human Brain Mapping, vol. 14, no. 3, pp. 140-151, 2001.

[23] R. L. Buckner, J. R. Andrews-Hanna, and D. L. Schacter, “The brain's default network: anatomy, function, and relevance to disease," Annals of the New York Academy of Sciences, vol. 1124, no. 1, pp. 1-38, 2008.

[24] M. E. Raichle, A. M. MacLeod, A. Z. Snyder, W. J. Powers, D. A. Gusnard, and G. L. Shulman, "A default mode of brain function," Proceedings of the National Academy of Sciences of the United States of America, vol. 98, no. 2, pp. 676-682, 2001.

[25] W. Liao, H. Chen, Y. Feng et al., "Selective aberrant functional connectivity of resting state networks in social anxiety disorder," NeuroImage, vol. 52, no. 4, pp. 1549-1558, 2010.

[26] M. Xia, J. Wang, and Y. He, "BrainNet viewer: a network visualization tool for human brain connectomics," PLOS ONE, vol. 8, no. 7, Article ID e68910, 2013.

[27] Z. X. Lv, D. H. Huang, W. Ye, Z. R. Chen, W. L. Huang, and J. O. Zheng, "Alteration of functional connectivity within visuospatial working memory-related brain network in patients with right temporal lobe epilepsy: a resting-state fMRI study," Epilepsy and Behavior, vol. 35, pp. 64-71, 2014.

[28] C. Sorg, V. Riedl, M. Mühlau et al., "Selective changes of restingstate networks in individuals at risk for Alzheimer's disease," Proceedings of the National Academy of Sciences of the United States of America, vol. 104, no. 47, pp. 18760-18765, 2007.

[29] J. M. Huang, C. M. Cheng, C. C. Chou, Y. C. Chen, P. Y. Hsu, and T. C. Yeh, "Physiological interference in effective connectivity of action network," Brain Imaging, vol. 24, no. 1, pp. 1-5, 2013.

[30] L. Schilbach, S. B. Eickhoff, A. Rotarska-Jagiela, G. R. Fink, and K. Vogeley, "Minds at rest? Social cognition as the default mode of cognizing and its putative relationship to the "default system" of the brain," Consciousness and Cognition, vol. 17, no. 2, pp. 457467, 2008.

[31] X.-N. Zuo, C. Kelly, J. S. Adelstein, D. F. Klein, F. X. Castellanos, and M. P. Milham, "Reliable intrinsic connectivity networks: test-retest evaluation using ICA and dual regression approach," NeuroImage, vol. 49, no. 3, pp. 2163-2177, 2010.

[32] N. B. Danielson, J. N. Guo, and H. Blumenfeld, "The default mode network and altered consciousness in epilepsy," Behavioural Neurology, vol. 24, no. 1, pp. 55-65, 2011.

[33] B. P. Kay, M. W. Difrancesco, M. D. Privitera, J. Gotman, S. K. Holland, and J. P. Szaflarski, "Reduced default mode network connectivity in treatment-resistant idiopathic generalized epilepsy," Epilepsia, vol. 54, no. 3, pp. 461-470, 2013.

[34] M. Song, H. Du, N. Wu et al., "Impaired resting-state functional integrations within default mode network of generalized tonicclonic seizures epilepsy," PLoS ONE, vol. 6, no. 2, Article ID e17294, 2011.

[35] W. Liao, Z. Zhang, Z. Pan et al., "Default mode network abnormalities in mesial temporal lobe epilepsy: a study combining fMRI and DTI," Human Brain Mapping, vol. 32, no. 6, pp. 883895, 2011.
[36] Z. Zhang, G. Lu, Y. Zhong et al., "Altered spontaneous neuronal activity of the default-mode network in mesial temporal lobe epilepsy," Brain Research, vol. 1323, pp. 152-160, 2010.

[37] A. C. Grant, K. M. Donnelly, C. Chubb, W. B. Barr, R. Kuzniecky, and O. Devinsky, "Temporal lobe epilepsy does not impair visual perception," Epilepsia, vol. 49, no. 4, pp. 710-713, 2008.

[38] T. Yang, C. Luo, Q. Li et al., "Altered resting-state connectivity during interictal generalized spike-wave discharges in drugnaive childhood absence epilepsy," Human Brain Mapping, vol. 34, no. 8, pp. 1761-1767, 2013.

[39] Z. Haneef, A. Lenartowicz, H. J. Yeh, J. Engel, and J. M. Stern, "Effect of lateralized temporal lobe epilepsy on the default mode network," Epilepsy and Behavior, vol. 25, no. 3, pp. 350-357, 2012.

[40] D. Sridharan, D. J. Levitin, and V. Menon, "A critical role for the right fronto-insular cortex in switching between centralexecutive and default-mode networks," Proceedings of the National Academy of Sciences of the United States of America, vol. 105, no. 34, pp. 12569-12574, 2008.

[41] R. Li, S. Wang, L. Zhu et al., "Aberrant functional connectivity of resting state networks in transient ischemic attack," PLoS ONE, vol. 8, no. 8, Article ID e71009, 2013.

[42] A. D’Argembeau, F. Collette, M. van der Linden et al., "Selfreferential reflective activity and its relationship with rest: a PET study," NeuroImage, vol. 25, no. 2, pp. 616-624, 2005.

[43] D. Mantini, M. G. Perrucci, C. Del Gratta, G. L. Romani, and M. Corbetta, "Electrophysiological signatures of resting state networks in the human brain," Proceedings of the National Academy of Sciences of the United States of America, vol. 104, no. 32, pp. 13170-13175, 2007.

[44] R. Qi, L. J. Zhang, Q. Xu et al., "Selective impairments of restingstate networks in minimal hepatic encephalopathy," PLoS ONE, vol. 7, no. 5, Article ID e37400, 2012.

[45] F. Bai, Y. Shi, Y. Yuan et al., "Altered self-referential network in resting-state amnestic type mild cognitive impairment," Cortex, vol. 48, no. 5, pp. 604-613, 2012.

[46] M. D. Greicius, B. H. Flores, V. Menon et al., "Restingstate functional connectivity in major depressionn: abnormally increased contributions from subgenual cingulate cortex and thalamus," Biological Psychiatry, vol. 62, no. 5, pp. 429-437, 2007.

[47] G. Bettus, E. Guedj, F. Joyeux et al., "Decreased basal fMRI functional connectivity in epileptogenic networks and contralateral compensatory mechanisms," Human Brain Mapping, vol. 30, no. 5, pp. 1580-1591, 2009.

[48] A. C. Coan, S. Appenzeller, L. M. Li, and F. Cendes, "Seizure frequency and lateralization affect progression of atrophy in temporal lobe epilepsy," Neurology, vol. 73, no. 11, pp. 834-842, 2009.

[49] A. Otti, H. Guendel, P. Henningsen, C. Zimmer, A. M. Wohlschlaeger, and M. Noll-Hussong, "Functional network connectivity of pain-related resting state networks in somatoform pain disorder: an exploratory fMRI study," Journal of Psychiatry \& Neuroscience, vol. 38, no. 1, pp. 57-65, 2013. 


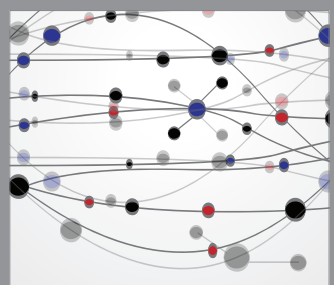

The Scientific World Journal
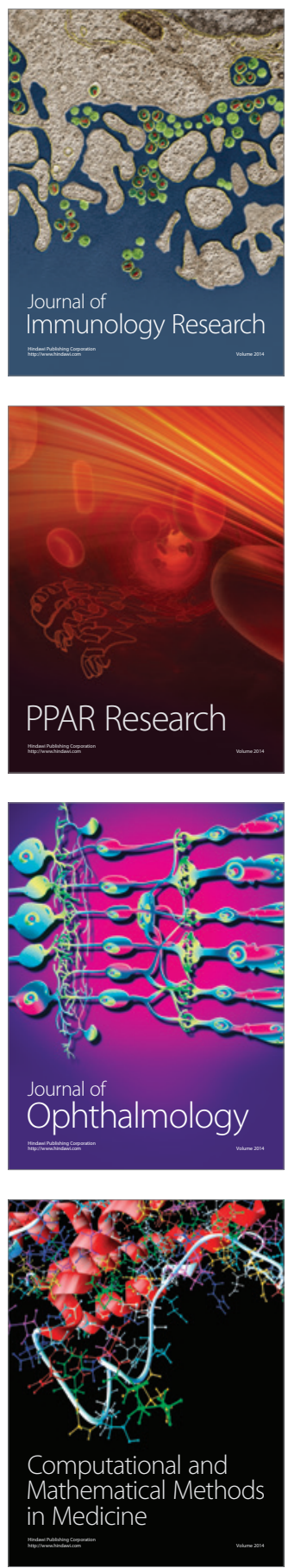

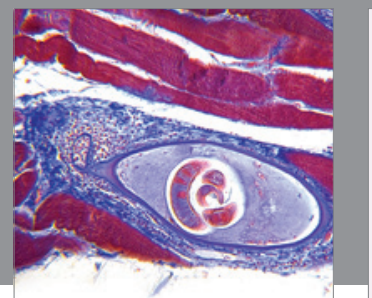

Gastroenterology

Research and Practice
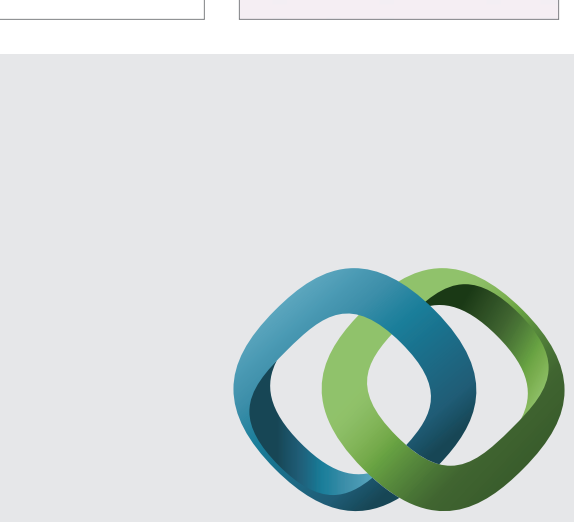

\section{Hindawi}

Submit your manuscripts at

http://www.hindawi.com
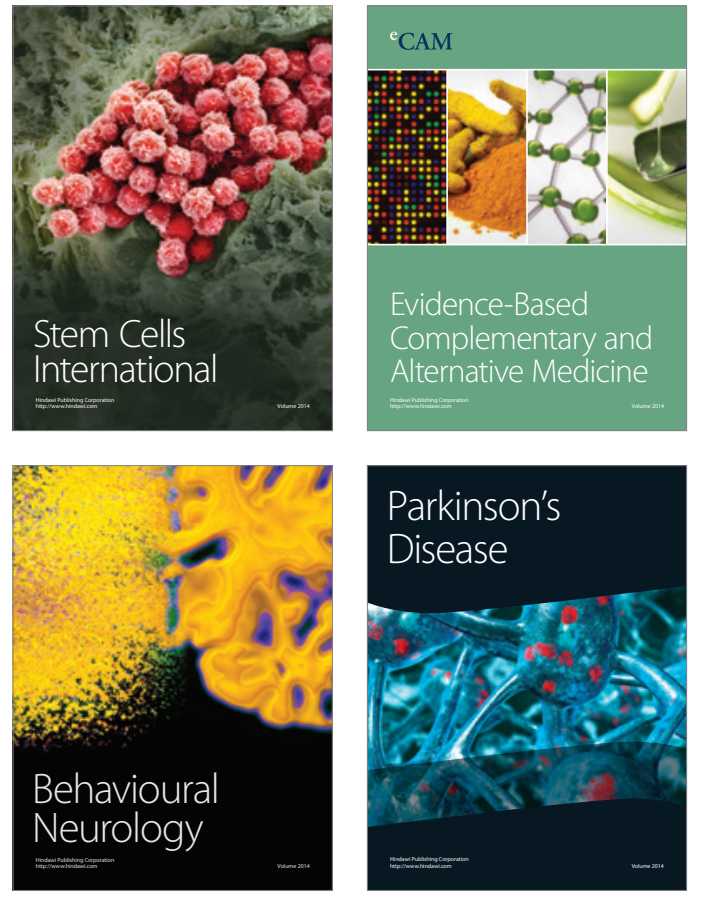
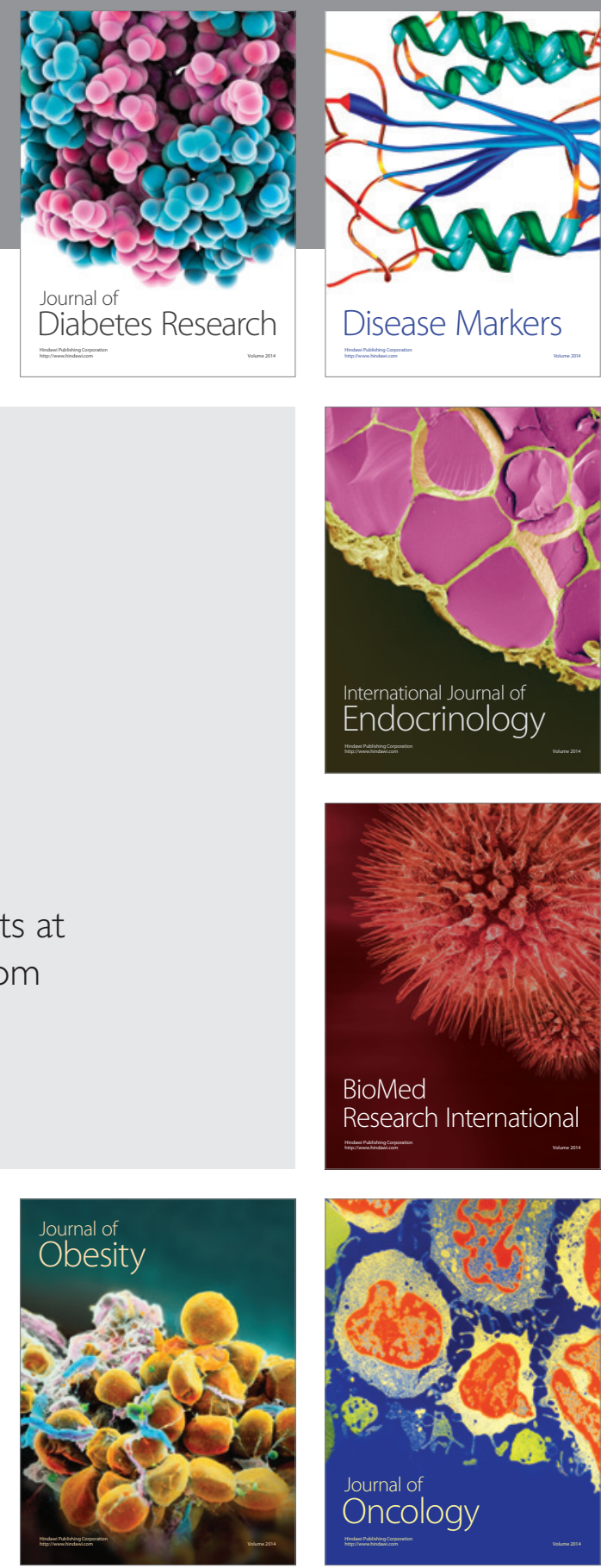

Disease Markers
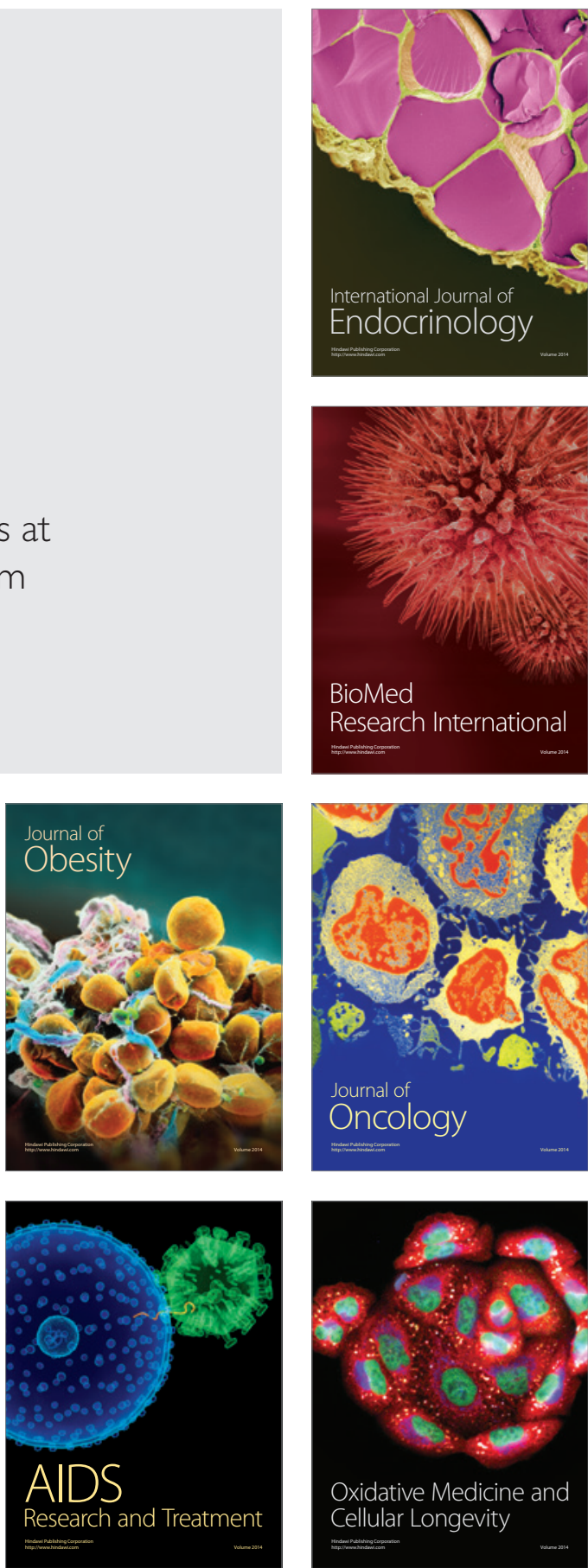